TRANSACTIONS OF THE

AMERICAN MATHEMATICAL SOCIETY

Volume 350, Number 7, July 1998, Pages 2939-2951

S 0002-9947(98)01969-2

\title{
A PROBABILISTIC APPROACH TO SOME OF EULER'S NUMBER THEORETIC IDENTITIES
}

\author{
DON RAWLINGS
}

\begin{abstract}
Probabilistic proofs and interpretations are given for the $q$-binomial theorem, $q$-binomial series, two of Euler's fundamental partition identities, and for $q$-analogs of product expansions for the Riemann zeta and Euler phi functions. The underlying processes involve Bernoulli trials with variable probabilities. Also presented are several variations on the classical derangement problem inherent in the distributions considered.
\end{abstract}

\section{INTRODUCTION}

For an integer $k \geq 1$, the $q$-shifted factorial of $t$ is defined as

$$
(t ; q)_{k}=(1-t)(1-t q) \cdots\left(1-t q^{k-1}\right) .
$$

By convention, $(t ; q)_{0}=1$. Using arguments on partitions, Euler showed that

$$
\sum_{k=0}^{\infty} \frac{q^{\left(\begin{array}{c}
k \\
2
\end{array}\right) t^{k}}}{(q ; q)_{k}}=\prod_{i=1}^{\infty}\left(1+t q^{i-1}\right)
$$

and

$$
\sum_{k=0}^{\infty} \frac{t^{k}}{(q ; q)_{k}}=\prod_{i=1}^{\infty}\left(1-t q^{i-1}\right)^{-1}
$$

hold formally. Identity (1) follows from the observation that the coefficient of $t^{k} q^{n-k}$ in either the sum or the product is equal to the number of partitions of $n$ into $k$ distinct parts. The coefficient of $t^{k} q^{n-k}$ in either side of (2) is the number of partitions of $n$ with $k$ parts (repetition allowed).

For $n \geq 0$, similar arguments on partitions with bounded parts yield the

q-Binomial Theorem: $(t ; q)_{n}=\sum_{k \geq 0}(-1)^{k} q^{\left(\begin{array}{l}k \\ 2\end{array}\right)}\left[\begin{array}{l}n \\ k\end{array}\right] t^{k}$

and the

q-Binomial Series: $1 /(t ; q)_{n+1}=\sum_{k \geq 0}\left[\begin{array}{c}n+k \\ k\end{array}\right] t^{k}$

Received by the editors August 8, 1996 and, in revised form, September 16, 1996.

1991 Mathematics Subject Classification. Primary 60K99, 11P81, 05A30, 05 A17.

Key words and phrases. Euler's process, Euler's partition identities, $q$-binomial theorem, $q$ Poisson distribution, $q$-derangement problem, $q$-Riemann zeta function, $q$-Euler phi function. 
where $\left[\begin{array}{l}n \\ k\end{array}\right]=\left(q^{n-k+1} ; q\right)_{k} /(q ; q)_{k}$ is the $q$-binomial coefficient.

Analytically, all four identities hold for $|t|,|q|<1$. For $|q|<1$, note that

$$
\lim _{n \rightarrow \infty}\left[\begin{array}{l}
n \\
k
\end{array}\right]=\lim _{n \rightarrow \infty} \frac{\left(1-q^{n}\right)\left(1-q^{n-1}\right) \cdots\left(1-q^{n-k+1}\right)}{(1-q)\left(1-q^{2}\right) \cdots\left(1-q^{k}\right)}=1 /(q ; q)_{k} .
$$

Thus, (1) and (2) follow as respective limits of the $q$-binomial theorem and series. A detailed account of these identities may be found in Andrews [1].

All four may also be deduced from probabilistic considerations. Kadell [10] has given just such a proof of the $q$-binomial series. Using the theory of complex variables, he then extended the $q$-binomial series to obtain a theorem due to Cauchy (see (2.2.1) in [1]) from which the other three follow as corollaries. Kadell similarly derived Ramanujan's ${ }_{1} \psi_{1}$ sum.

Another probabilistic approach is presented herein. The geometry of the underlying processes exposes a connection with the derangement problem of combinatorics and leads to $q$-analogs of classical product expansions for the Riemann zeta and Euler phi functions. The derangement problem presented in section 4 extends the $q$-derangement problem solved by Wachs [16].

The basic model, referred to as Euler's process, is as follows. For the first column of an $n \times n$ array of cells select a solid dot $\bullet$ with probability $t$ or a clear dot $\odot$ with probability $(1-t)$, where $0 \leq t \leq 1$. The selected dot is placed in the bottom cell of column 1 and a coin with probability $q<1$ of landing tails up is tossed until heads occurs. Each time tails occurs, the dot moves up a cell with one exception: If tails occurs when the dot is in the top cell, then the dot moves back to the bottom cell. When heads occurs, the dot comes to rest. The procedure for column 1 is successively repeated for columns 2 through $n$. For $n=3$, the outcome for a given selection of dots with associated Bernoulli sequences is illustrated below.

\section{Example 1.}
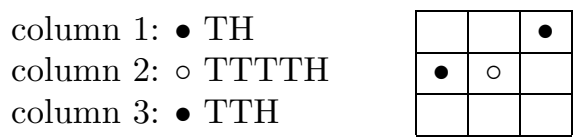

The symbol $\mathcal{D}$ will be used to denote the number of solid dots that come to rest on the diagonal running from the lower left corner to the upper right corner. In Example $1, \mathcal{D}=1$.

Several distributions relative to Euler's process and some of its variations are computed in sections 2 through 6 . In section 2, the distribution of $\mathcal{D}$ for Euler's process is shown to asymptotically approach a distribution best described as being $q$-Poisson. Immediate consequences include the $q$-binomial theorem and (1). The variation of Euler's process found in section 3 leads to a second $q$-Poisson distribution, and to probabilistic proofs of the $q$-binomial series and of (2). The distributions in sections 5 and 6 imply the aforementioned $q$-identities for the Riemann zeta and Euler phi functions.

\section{A DiAgonal Distribution-NO REPEATED Hits}

Euler's process allows at most one hit per cell (i.e., at most one dot comes to rest in any given cell). To determine the distribution of $\mathcal{D}$, two preliminary facts are needed. First, with the rows numbered 1 through $n$ from bottom to top, the 
probability of a solid dot coming to rest in row $i$ is

$$
t \sum_{k=0}^{\infty} q^{i-1+k n}(1-q)=t(1-q) q^{i-1} \sum_{k=0}^{\infty}\left(q^{n}\right)^{k}=\frac{t q^{i-1}}{[n]}
$$

where $[n]=\left(1-q^{n}\right) /(1-q)$. Second, the generating function for partitions with $k$ parts, each bounded by $m$, is known $[1$, p. 33$]$ to be

$$
\sum_{1 \leq \lambda_{1} \leq \lambda_{2} \leq \ldots \leq \lambda_{k} \leq m} q^{\lambda_{1}+\lambda_{2}+\cdots+\lambda_{k}}=q^{k}\left[\begin{array}{c}
m+k-1 \\
k
\end{array}\right] .
$$

The distribution of $\mathcal{D}$ and its asymptotic limit are given by

Theorem 1. For Euler's process with $0 \leq q<1$ and $0 \leq t \leq 1, k$ solid dots come to rest on the diagonal of an $n \times n$ array of cells with probability

$$
P_{n}\{\mathcal{D}=k\}=\sum_{j=k}^{n}(-1)^{j-k}\left(\begin{array}{l}
j \\
k
\end{array}\right) q^{\left(\begin{array}{c}
j \\
2
\end{array}\right)}\left[\begin{array}{l}
n \\
j
\end{array}\right] \frac{t^{j}}{[n]^{j}} .
$$

As $n \rightarrow \infty$, the distribution of $\mathcal{D}$ approaches

$$
P\{\mathcal{D}=k\}=\sum_{j=k}^{\infty}(-1)^{j-k}\left(\begin{array}{l}
j \\
k
\end{array}\right) q^{\left(\begin{array}{c}
j \\
2
\end{array}\right)} \frac{t^{j}}{[j] !},
$$

where $[j] !=[1][2] \cdots[j]$ for $j \geq 1$ and $[0] !=1$.

Proof. Let $A_{\mu}$ denote the event that the $\mu^{\text {th }}$ dot selected is solid and comes to rest on the diagonal. For $1 \leq \mu_{1}<\mu_{2}<\ldots<\mu_{j} \leq n$, (4) implies that the probability of $A_{\mu_{1}} \cap A_{\mu_{2}} \cap \cdots \cap A_{\mu_{j}}$ occurring is $t^{k} q^{\mu_{1}+\mu_{2}+\cdots+\mu_{j}-j} /[n]^{j}$. By Whitworth's [3, p. 102] extension of the principle of inclusion-exclusion,

$$
\begin{aligned}
P_{n}\{\mathcal{D}=k\} & =\sum_{j=k}^{n}(-1)^{j-k}\left(\begin{array}{l}
j \\
k
\end{array}\right) \sum_{1 \leq \mu_{1}<\ldots<\mu_{j} \leq n} P_{n}\left\{A_{\mu_{1}} \cap \cdots \cap A_{\mu_{j}}\right\} \\
& =\sum_{j=k}^{n}(-1)^{j-k}\left(\begin{array}{l}
j \\
k
\end{array}\right) \frac{t^{j} q^{-j}}{[n]^{j}} \sum_{1 \leq \mu_{1}<\ldots<\mu_{j} \leq n} q^{\mu_{1}+\cdots+\mu_{j}} \\
& =\sum_{j=k}^{n}(-1)^{j-k}\left(\begin{array}{l}
j \\
k
\end{array}\right) \frac{t^{j} q^{-j} q^{\left(\begin{array}{c}
j \\
2
\end{array}\right)}}{[n]^{j}} \sum_{1 \leq \lambda_{1} \leq \ldots \leq \lambda_{j} \leq n-j+1} q^{\lambda_{1}+\cdots+\lambda_{j}} .
\end{aligned}
$$

The above calculation together with (5) implies (6). Formula (7) follows from (6), $(3)$, and the fact that $\lim _{n \rightarrow \infty}[n]=1 /(1-q)$.

Both the $q$-binomial theorem and Euler's identity (1) are easily deduced from Theorem 1: Since a solid dot comes to rest in the $i^{\text {th }}$ diagonal cell of an $n \times n$ array with probability $t q^{i-1} /[n]$, it is clear by inspection that the probability of no solid dots coming to rest on the diagonal is

$$
P_{n}\{\mathcal{D}=0\}=\prod_{i=1}^{n}\left(1-t q^{i-1} /[n]\right)=(t /[n] ; q)_{n} .
$$

From (6), we also have

$$
P_{n}\{\mathcal{D}=0\}=\sum_{j=0}^{n}(-1)^{j} q^{\left(\begin{array}{c}
j \\
2
\end{array}\right)}\left[\begin{array}{l}
n \\
j
\end{array}\right] \frac{t^{j}}{[n]^{j}}
$$


Equating the last two expressions for $P_{n}\{\mathcal{D}=0\}$ and replacing $t$ by $t[n]$ gives the $q$-binomial theorem. Similary, (1) follows from the observation that

$$
P\{\mathcal{D}=0\}=\lim _{n \rightarrow \infty} \prod_{i=1}^{n}\left(1-t q^{i-1} /[n]\right)=\prod_{i=1}^{\infty}\left(1-t q^{i-1}(1-q)\right)
$$

combined with (7) and the replacement of $t$ by $t /(q-1)$.

Remark 1. Define "success" to mean that a solid dot comes to rest on the diagonal. Further, let $p_{i}$ denote the probability of a solid dot hitting the diagonal of the $i^{\text {th }}$ column. Then, (6) is just the success distribution for Bernoulli trials with the variable success probabilities $\left[8\right.$, p. 282] $p_{1}=t /[n], p_{2}=t q /[n], \ldots, p_{n}=t q^{n-1} /[n]$. The probability generating function is $\sum_{k=0}^{n} P_{n}\{\mathcal{D}=k\} z^{k}=\prod_{i=1}^{n}\left(1-t(1-z) q^{i-1} /[n]\right)$. In general, the distribution associated with Euler's process hitting any given set $\mathcal{C}$ of cells amounts to Bernoulli trials with variable probabilities. For $\mathcal{C}$ consisting of the diagonal unioned with the first row, the probabilities by column of hitting $\mathcal{C}$ are $p_{1}=t /[n], p_{2}=t(1+q) /[n], \ldots, p_{n}=t\left(1+q^{n-1}\right) /[n]$.

Remark 2. As $q \rightarrow 1^{-},(7)$ tends to a Poisson distribution:

$$
\lim _{q \rightarrow 1^{-}} P\{\mathcal{D}=k\}=\sum_{j=k}^{\infty}(-1)^{j-k}\left(\begin{array}{l}
j \\
k
\end{array}\right) \frac{t^{j}}{j !}=\frac{t^{k}}{k !} \sum_{j=k}^{\infty} \frac{(-1)^{j-k} t^{j-k}}{(j-k) !}=\frac{t^{k} e^{-t}}{k !} .
$$

Thus, (7) may aptly be described as being $q$-Poisson with parameter $t$.

Remark 3. Theorem 1 affords a solution to a variation on the classical derangement problem. In the event that the $i^{t h}$ dot selected is solid (respectively, clear), let $F(i)$ be the row number (respectively, the row number underlined) in which the $i^{t h} \operatorname{dot}$ comes to rest. The mapping $F=F(1) F(2) \ldots F(n)$ encodes the outcome of Euler's process. A $k$ such that $F(k)=k$ is said to be a fixed point of $F$. In Example $1, F=2 \underline{2} 3$ has one fixed point. From (7), the asymptotic probability of Euler's process generating a mapping with no fixed points is

$$
\lim _{n \rightarrow \infty} P_{n}\{F(i) \neq i, 1 \leq i \leq n\}=P\{\mathcal{D}=0\}=E_{q}(-t)
$$

where $E_{q}(t)=\sum_{j=0}^{\infty} q^{\left(\begin{array}{c}j \\ 2\end{array}\right)} t^{j} /[j]$ ! is a $q$-analog of the exponential function (satisfying $\left.\lim _{q \rightarrow 1^{-}} E_{q}(t)=e^{t}\right)$. Putting $t=1$ in (8) gives a result presented in [13].

\section{A DiAgOnAl Distribution-REPEATED Hits}

A slight modification of Euler's process leads to probabilistic proofs of the $q$ binomial series and of (2). Referred to as Euler's negative q-binomial process, the modified procedure consists of selecting and Bernoulli propelling a sequence of dots up each column of an $n \times n$ array. For $1 \leq i \leq n$, the sequence in column $i$ is terminated after a clear dot is selected or after a solid dot fails to stop on the diagonal cell. Example 2 provides an illustration.

\section{Example 2.}

$$
\begin{aligned}
& \text { column 1: } \bullet \text { TTTH, ० H } \\
& \text { column 2: } \bullet \text { TTH } \\
& \text { column 3: } \bullet \text { TTH, } \bullet \text { TTH, 。 TH }
\end{aligned}
$$

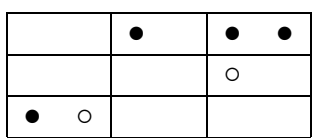


Note that $\mathcal{D}=3$ solid dots have come to rest on the diagonal in the example above. The following result holds.

Theorem 2. For Euler's negative $q$-binomial process with $0 \leq q<1$ and $0 \leq t \leq 1$, $k$ solid dots come to rest on the diagonal of an $n \times n$ array of cells with probability

$$
Q_{n}\{\mathcal{D}=k\}=(t /[n] ; q)_{n}\left[\begin{array}{c}
n+k-1 \\
k
\end{array}\right] \frac{t^{k}}{[n]^{k}} .
$$

As $n \rightarrow \infty$, the distribution of $\mathcal{D}$ approaches

$$
Q\{\mathcal{D}=k\}=\frac{t^{k}}{[k] !} \prod_{i=1}^{\infty}\left(1-t q^{i-1}(1-q)\right) .
$$

Proof. In view of (4), the probability of exactly $\mu_{i} \geq 0$ solid dots coming to rest in the diagonal cell of the $i^{t h}$ column is $\left(t q^{i-1} /[n]\right)^{\mu_{i}}\left(1-t q^{i-1} /[n]\right)$. Thus, a total of $k$ solid dots stop on the diagonal with probability

$$
Q_{n}\{\mathcal{D}=k\}=\frac{t^{k}}{[n]^{k}} \prod_{i=1}^{n}\left(1-t q^{i-1} /[n]\right) \sum_{\mu_{1}+\mu_{2}+\cdots+\mu_{n}=k} q^{\mu_{2}+2 \mu_{3}+\cdots+(n-1) \mu_{n}} .
$$

By setting $\lambda_{i}=1+\mu_{n-i+1}+\mu_{n-i+2}+\cdots+\mu_{n}$ for $1 \leq i \leq(n-1)$ and making use of (5), the preceding equality can be rewritten to give (9):

$$
\begin{aligned}
Q_{n}\{\mathcal{D}=k\} & =\frac{t^{k}}{[n]^{k}}(t /[n] ; q)_{n} \sum_{1 \leq \lambda_{1} \leq \ldots \leq \lambda_{n-1} \leq k+1} q^{\lambda_{1}+\cdots+\lambda_{n-1}-(n-1)} \\
& =(t /[n] ; q)_{n}\left[\begin{array}{c}
n+k-1 \\
n-1
\end{array}\right] \frac{t^{k}}{[n]^{k}} .
\end{aligned}
$$

Formula (10) is easily deduced from (9) and (3).

The $q$-binomial series and (2) follow almost immediately from Theorem 2. Since $Q_{n}$ is a measure, (9) implies that

$$
1=\sum_{k \geq 0} Q_{n}\{\mathcal{D}=k\}=(t /[n] ; q)_{n} \sum_{k \geq 0}\left[\begin{array}{c}
n+k-1 \\
k
\end{array}\right] \frac{t^{k}}{[n]^{k}} .
$$

Dividing through by $(t /[n] ; q)_{n}$ and replacing $t$ by $t[n]$ gives the $q$-binomial series. Similarly, (2) is deduced from $1=\sum_{k \geq 0} Q\{\mathcal{D}=k\}$ and (10).

Remark 4. The distributions of Theorem 2 and their relatives arise in the oil drilling strategies of Benkerhouf and Bather [2] and, as surveyed by Kemp [11], in studying parasites on hosts, elective queues, and in many other applications. Often, $Q\{\mathcal{D}=\cdot\}$ is referred to as a Heine distribution. From $[9,11]$ or the $q$-binomial series, the probability generating function for (9) is

$$
\sum_{k \geq 0} Q_{n}\{\mathcal{D}=k\} z^{k}=\prod_{i=1}^{n}\left(\frac{1-t q^{i-1} /[n]}{1-z t q^{i-1} /[n]}\right) .
$$


Remark 5. As noted in [9, 11], (10) is also $q$-Poisson. To see this, consider the $q$-exponential function defined by $e_{q}(t)=\sum_{k \geq 0} t^{k} /[k]$ !. Replacing $t$ by $t(1-q)$ in (2) shows that $e_{q}(t)=\prod_{i \geq 1}\left(1-t q^{i-1}(1-q)\right)^{-1}$. Thus, (10) has the alternate form

$$
Q\{\mathcal{D}=k\}=\frac{t^{k}}{[k] ! e_{q}(t)}
$$

and it follows that $\lim _{q \rightarrow 1^{-}} Q\{\mathcal{D}=k\}=t^{k} e^{-t} / k !$.

Remark 6. Let $F$ be as in Remark 3. For Example 2, $F=11333 \underline{2}$ has two fixed points. From Remark 5, the asymptotic probability of Euler's negative $q$-binomial distribution generating a mapping with no fixed points is

$$
\lim _{n \rightarrow \infty} Q_{n}\{F(i) \neq i, 1 \leq i \leq n\}=Q\{\mathcal{D}=0\}=1 / e_{q}(t) .
$$

Remark 7. As generators of mappings with no fixed points, Euler's process and Euler's negative $q$-binomial process are indistinguishable. Thus, (8) and (11) give a probabilistic explanation of the known identity $E_{q}(-t)=1 / e_{q}(t)$.

\section{A $(t, q)$-DERANGEMENT PROBlem}

The comajor index process is Euler's process altered by deleting rows as they are occupied and by propelling the $(i+1)^{s t}$ dot from the next available row above where the $i^{\text {th }}$ dot comes to rest. Using x's to mark deleted cells, Example 3 serves as a clarification.

\begin{tabular}{|c|c|c|c|c|}
\hline column 1: • TTH & & & $\bullet$ & $\mathrm{x}$ \\
\hline column 2: ○ TTH & $\bullet$ & $\mathrm{x}$ & $\mathrm{x}$ & $\mathrm{x}$ \\
\hline column $3: \bullet \mathrm{H}$ & & o & $\mathrm{x}$ & $\mathrm{x}$ \\
\hline column 4: ○ $\mathrm{TH}$ & & & & o \\
\hline
\end{tabular}

\section{Example 3.}

For $n \geq 1$, let $F$ be as in Remark 3 and define $\Theta=\Theta(1) \Theta(2) \ldots \Theta(n)$ by setting $\Theta(i)$ equal to the row number where the dot in the $i^{\text {th }}$ column comes to rest. Thus, $\Theta$ maps the set of outcomes to the set of permutations $S_{n}$ on $\{1,2, \ldots, n\}$. In Example 3, $F=3 \underline{2} 4 \underline{1}$ and $\Theta=3241 \in S_{4}$. Further, let $\mathcal{D}$ be as in section 1 and define $\mathcal{D}_{\Theta}$ to be the total number of dots that stop on the diagonal. The values of $\mathcal{D}$ and $\mathcal{D}_{\Theta}$ correspond to the respective numbers of fixed points of $F$ and $\Theta$. In Example $3, \mathcal{D}=0$ and $\mathcal{D}_{\Theta}=1$.

The comajor index of a permutation $\theta \in S_{n}$ is defined to be

$$
\operatorname{comaj} \theta=\sum_{k}(n-k),
$$

where the sum is over all $k$ satisfying $1 \leq k<n$ and $\theta(k)>\theta(k+1)$. Rawlings and Treadway [15] showed that the probability of a given permutation $\theta \in S_{n}$ being generated by the comajor index process is

$$
C_{n}\{\Theta=\theta\}=q^{\operatorname{comaj} \theta} /[n] !
$$

In slightly different terms, (12) was first obtained by Moritz and Williams [12].

From (12), we see that $C_{n}\{\Theta=\cdot\}$ is a $q$-analog of the uniform measure on $S_{n}$ : $\lim _{q \rightarrow 1^{-}} C_{n}\{\Theta=\theta\}=1 / n$ ! . Thus, determining the value of $\lim _{n \rightarrow \infty} C_{n}\left\{\mathcal{D}_{\Theta}=0\right\}$ amounts to solving a $q$-analog of the usual derangement problem. As will be seen, Wachs [16] has shown this limit to be $E_{q}(-1)$. 
In view of sections 2 and 3 , it is natural to consider the $(t, q)$-derangement problem of finding $\lim _{n \rightarrow \infty} C_{n}\{\mathcal{D}=0\}$. To obtain a tractable answer, the probability of selecting a solid dot is varied from column to column by the number of clear dots on the diagonal: For $0 \leq q<1$ and $0 \leq t \leq 1$, let

$$
t_{i}=t q^{|\{F(j)=\underline{j}: 1 \leq j<i\}|}
$$

be the probability of selecting a solid dot for column $i$. Of course, the corresponding probability of selecting a clear dot is $\left(1-t_{i}\right)$. In Example $3, t_{1}=t_{2}=t$ and $t_{3}=t_{4}=t q$. Formula (14) below is a minor reformulation of a result obtained by Wachs [16].

Theorem 3. For the comajor index process with $0 \leq q<1$ and $t_{i}$ as in (13), the fixed point distributions of $\Theta$ and $F$ on an $n \times n$ array are respectively

$$
C_{n}\left\{\mathcal{D}_{\Theta}=k\right\}=\frac{1}{[k] !} \sum_{j=0}^{n-k} \frac{(-1)^{j} q^{\left(\begin{array}{c}
j \\
2
\end{array}\right)}}{[j] !}
$$

and

$$
C_{n}\{\mathcal{D}=k\}=\frac{t^{k}}{[k] !} \sum_{j=0}^{n-k} \frac{(-1)^{j} q^{\left(\begin{array}{c}
j \\
2
\end{array}\right) t^{j}}}{[j] !} .
$$

As $n \rightarrow \infty$, (15) tends to a q-Poisson distribution with parameter $t:$

$$
C\{\mathcal{D}=k\}=\frac{t^{k} E_{q}(-t)}{[k] !}
$$

Proof. For convenience, let $c_{n, k}$ denote the lefthand side of (14) and let

$$
d_{n, k}=\sum_{\theta} q^{\operatorname{comaj} \theta}
$$

where the sum is over all $\theta \in S_{n}$ with exactly $k$ fixed points. In view of (12), we have $c_{n, k}=d_{n, k} /[n]$ !. From [16] or from formula (8.6) of Gessel and Reutenauer [6], it is not difficult to deduce that

$$
d_{n, k}=\left[\begin{array}{c}
n \\
k
\end{array}\right] d_{n-k, 0}=\frac{[n] !}{[k] !} \sum_{j=0}^{n-k} \frac{(-1)^{j} q^{\left(\begin{array}{c}
j \\
2
\end{array}\right)}}{[j] !} .
$$

This together with the fact that $c_{n, k}=d_{n, k} /[n]$ ! establishes (14) and implies the alternate formula

$$
c_{n, k}=\frac{1}{[n] !}\left[\begin{array}{l}
n \\
k
\end{array}\right] d_{n-k, 0}=\frac{d_{n-k, 0}}{[n-k] ![k] !} .
$$

To establish (15), first observe that

$$
1=\sum_{k=0}^{n} c_{n, k}=\sum_{k=0}^{n} \frac{d_{n-k, 0}}{[n-k] ![k] !} .
$$

Next, suppose that $l$ dots come to rest on the diagonal. For $\mathcal{D}$ to equal $k$, it must be the case that $l \geq k$ and that exactly $k$ of the $l$ diagonal dots are solid. Letting $\mu_{i}$ indicate the number of solid diagonal dots lying between the $i^{\text {th }}$ and $(i+1)^{s t}$ clear diagonal dots, it follows that

$$
C_{n}\{\mathcal{D}=k\}=\sum_{l=k}^{n} c_{n, l}(t ; q)_{l-k} \sum_{\mu_{0}+\mu_{1}+\cdots+\mu_{l-k}=k} t^{k} q^{\mu_{1}+2 \mu_{2}+\cdots+(l-k) \mu_{l-k}} .
$$


Using (16), the $q$-binomial theorem, a substitition as in the proof of Theorem 2, an interchange in the order of summation, and finally (17) leads to

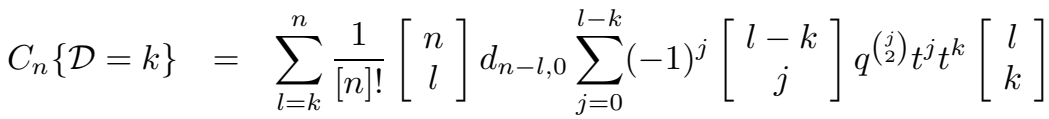

$$
\begin{aligned}
& =\frac{t^{k}}{[k] !} \sum_{j=0}^{n} \frac{(-1)^{j} q^{\left(\begin{array}{c}
j \\
2
\end{array}\right)} t^{j}}{[j] !} \sum_{l=j+k}^{n} \frac{d_{n-l, 0}}{[n-l] ![l-k-j] !} \\
& =\frac{t^{k}}{[k] !} \sum_{j=0}^{n-k} \frac{(-1)^{j} q^{\left(\begin{array}{c}
j \\
2
\end{array}\right)} t^{j}}{[j] !} \text {. }
\end{aligned}
$$

It follows from Theorem 3 that the asymptotic probability of the comajor index process generating a function with no fixed points is

$$
\lim _{n \rightarrow \infty} C_{n}\{F(i) \neq i, 1 \leq i \leq n\}=C\{\mathcal{D}=0\}=E_{q}(-t)
$$

For $t=1$, we have $t_{1}=\ldots=t_{n}=1$, and (18) reduces to the solution of the $q$-derangement problem resolved by Wachs [16]. Letting $q \rightarrow 1^{-}$leads to

Corollary 1. Define $S_{n}$ to be the set of functions generated by (a) first randomly selecting a permutation $\theta=\theta(1) \theta(2) \ldots \theta(n) \in S_{n}$ and (b) then, for $1 \leq i \leq n$, underlining $\theta(i)$ with probability $(1-t)$. As $n \rightarrow \infty$, the probability of generating a function in $\underline{S_{n}}$ having no fixed points tends to $e^{-t}$.

Remark 8. From Remarks 5 and 7, the $q$-Poisson distributions of Theorems 2 and 3 are seen to be identical.

Remark 9. The sequence $d_{n, k}$ and $q$-derangement numbers $d_{n, 0}$ have been considered in other contexts by Gessel [5] and by Garsia and Remmel [4]. A q-derangement problem relative to a process associated with the inversion number of a permutation was recently solved by Griffin [7].

\section{The Riemann Zeta Distribution}

Euler's process may also be modified to obtain a probabilistic proof of the $q$ analog in [13] for Euler's product formula of the Riemann zeta function. Let $p_{1}, p_{2}, \ldots, p_{m}$ be a listing of the smallest $m$ prime numbers in their natural order. Euler's process altered by

(a) replacing the square array of cells with an array having $m$ columns in which the $i^{\text {th }}$ column contains $p_{i}$ cells,

(b) varying the selection probabilities by column $\left(t_{i}\right.$ and $\left(1-t_{i}\right)$ will denote the probabilities of selecting a solid and clear dot in column $i)$, and

(c) selecting and Bernoulli propelling a sequence of dots up each column; the sequence in column $i$ being terminated after a clear dot is selected or after a solid dot fails to stop in the $p_{i}^{t h}$ (top) cell

will be referred to as the Riemann zeta process. As an illustration for $m=3$, we have 


\section{Example 4.}
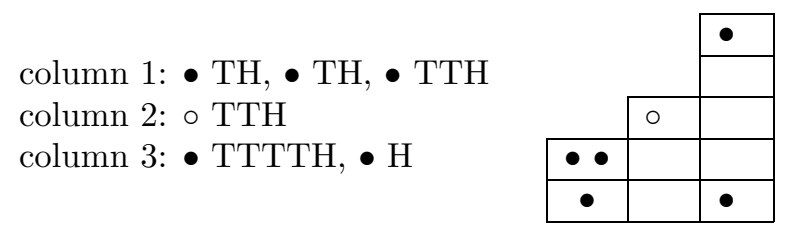

The symbol $\mathcal{N}$ will be used to denote the product $p_{1}^{\alpha_{1}} p_{2}^{\alpha_{2}} \cdots p_{m}^{\alpha_{m}}$, where $\alpha_{i}$ is the number of solid dots that come to rest in the top cell of column $i$. In Example 4, $\mathcal{N}=2^{2} 3^{0} 5^{1}=20$. Thus, the Riemann zeta process generates prime factorizations of integers. The distribution of $\mathcal{N}$ is given by

Theorem 4. For the Riemann zeta process on $m$ columns with $0 \leq q<1$ and $0 \leq t_{i} \leq 1$, the probability of $n=p_{1}^{\alpha_{1}} p_{2}^{\alpha_{2}} \cdots p_{m}^{\alpha_{m}}$ being generated is

$$
Z_{m}\{\mathcal{N}=n\}=\frac{1}{\|n\|} \prod_{i=1}^{m} t_{i}^{\alpha_{i}} q^{\alpha_{i}\left(p_{i}-1\right)}\left(1-t_{i} q^{p_{i}-1} /\left[p_{i}\right]\right),
$$

where $\|n\|=\left[p_{1}\right]^{\alpha_{1}}\left[p_{2}\right]^{\alpha_{2}} \cdots\left[p_{m}\right]^{\alpha_{m}}$.

Proof. From (4), it follows that the probability of exactly $\alpha_{i}$ solid dots stopping in the top cell of the $i^{\text {th }}$ column is $\left(t_{i} q^{p_{i}-1} /\left[p_{i}\right]\right)^{\alpha_{i}}\left(1-t_{i} q^{p_{i}-1} /\left[p_{i}\right]\right)$.

For $s>1$ and $0 \leq q<1$, let

$$
\zeta_{q}(s)=\sum_{n \geq 1} \frac{q^{s \nu(n)}}{\|n\|^{s}}
$$

where, by definition, $\nu(1)=1$ and, for $n=p_{1}^{\alpha_{1}} p_{2}^{\alpha_{2}} \cdots p_{m}^{\alpha_{m}}>1, \nu(n)=\alpha_{1}\left(p_{1}-1\right)+$ $\alpha_{2}\left(p_{2}-1\right)+\cdots+\alpha_{m}\left(p_{m}-1\right)$. Note that $\lim _{q \rightarrow 1^{-}} \zeta_{q}(s)$ is the usual Riemann zeta function $\zeta(s)$.

To obtain the advertised $q$-analog of Euler's classical identity

$$
\zeta(s)=\prod_{p \text { prime }}\left(1-1 / p^{s}\right)^{-1},
$$

first note that

$$
\sum_{n \in N_{m}} Z_{m}\{\mathcal{N}=n\}=1,
$$

where $N_{m}$ denotes the set of positive integers having only prime factors less than or equal to $p_{m}$. Then combining (19) with (20) leads to

$$
\sum_{n=p_{1}^{\alpha_{1}} p_{2}^{\alpha_{2} \cdots p_{m}^{\alpha_{m}} \in N_{m}}} \frac{q^{\nu(n)}}{\|n\|} \prod_{i=1}^{m} t_{i}^{\alpha_{i}}=\prod_{i=1}^{m}\left(1-t_{i} q^{p_{i}-1} /\left[p_{i}\right]\right)^{-1} .
$$

Finally, setting $t_{i}=\left(q^{p_{i}-1} /\left[p_{i}\right]\right)^{s-1}$ and taking the limit of $(21)$ as $m \rightarrow \infty$ gives a $q$-analog of Euler's product formula for the Riemann zeta function:

$$
\zeta_{q}(s)=\prod_{p \text { prime }}\left(1-q^{s(p-1)} /[p]^{s}\right)^{-1} .
$$

Convergence of $\zeta_{q}(s)$ and of the product for $s>1$ was established in [13]. 
Remark 10. By (21), the probability generating function for the number of primes is

$$
\sum_{n=p_{1}^{\alpha_{1}} p_{2}^{\alpha_{2} \ldots p_{m}^{\alpha_{m}} \in N_{m}}} Z_{m}\{\mathcal{N}=n\} z^{\alpha_{1}+\alpha_{2}+\cdots+\alpha_{m}}=\prod_{i=1}^{m}\left(\frac{1-t_{i} q^{p_{i}-1} /\left[p_{i}\right]}{1-z t_{i} q^{p_{i}-1} /\left[p_{i}\right]}\right) .
$$

Remark 11. If $t_{i}=\left(q^{p_{i}-1} /\left[p_{i}\right]\right)^{s-1}$, then the asymptotic probability of the Riemann zeta process generating the integer 1 is

$$
\lim _{m \rightarrow \infty} Z_{m}\{\mathcal{N}=1\}=\lim _{m \rightarrow \infty} \frac{q^{s \nu(1)}}{\|1\|^{s}} \prod_{i=1}^{m}\left(1-q^{s\left(p_{i}-1\right)} /\left[p_{i}\right]^{s}\right)=1 / \zeta_{q}(s) .
$$

Alternatively, $\lim _{m \rightarrow \infty} Z_{m}\left\{F(i) \neq p_{i}, 1 \leq i \leq m\right\}=1 / \zeta_{q}(s)$, where $F$ is as in Remark 3. Another interpretation of $1 / \zeta_{q}(s)$ was given in [13].

\section{The Euler Phi Process}

The value of the Euler phi function $\phi$ at $n \geq 1$ is defined to be the number of positive integers not exceeding $n$ that are relatively prime to $n$, that is,

$$
\phi(n)=|\{k: 1 \leq k \leq n, \operatorname{gcd}(k, n)=1\}| .
$$

The classic identity expressing $\phi(n)$ in terms of the prime divisors $p$ of $n$,

$$
\phi(n)=n \prod_{p \mid n}(1-1 / p),
$$

may also be derived and extended through probabilistic considerations. Two such approaches are considered herein. The first, undoubtly known, serves as a frame of reference for the second.

For $n \geq 1$, consider randomly selecting an integer $\mathcal{N}$ between 1 and $n$ (inclusive). The induced measure $U_{n}\{\cdot\}$ is uniform: $U_{n}\{\mathcal{N}=k\}=1 / n$ for $1 \leq k \leq n$. As $\phi(n) / n$ is the probability of randomly selecting an integer relatively prime to $n$ from the set $\{1,2, \ldots, n\}$, we have

$$
\phi(n) / n=U_{n}\{\operatorname{gcd}(\mathcal{N}, n)=1\} .
$$

Suppose that $n=r_{1}^{\alpha_{1}} r_{2}^{\alpha_{2}} \cdots r_{j}^{\alpha_{j}}$, where $r_{1}, r_{2}, \ldots, r_{j}$ are distinct primes and $\alpha_{1}, \alpha_{2}$, $\ldots, \alpha_{j} \geq 1$. For $1 \leq i_{1}<i_{2}<\ldots<i_{l} \leq j$, the integers divisible by $m=$ $r_{i_{1}} r_{i_{2}} \cdots r_{i_{l}}$ in $\{1,2, \ldots, n\}$ are $m, 2 m, \ldots,(n / m) m$. Thus,

$$
U_{n}\left\{r_{i_{\nu}} \mid \mathcal{N}, 1 \leq \nu \leq l\right\}=\frac{n / m}{n}=\frac{1}{r_{i_{1}} r_{i_{2}} \cdots r_{i_{l}}} .
$$

Hence, the events $r_{1}\left|\mathcal{N}, r_{2}\right| \mathcal{N}, \ldots, r_{j} \mid \mathcal{N}$ are independent and it follows that

$$
\begin{array}{r}
U_{n}\{\operatorname{gcd}(\mathcal{N}, n)=1\}=U_{n}\left\{r_{i} \backslash \mathcal{N}, 1 \leq i \leq j\right\} \\
=\prod_{i=1}^{j}\left(1-U_{n}\left\{r_{i} \mid \mathcal{N}\right\}\right)=\prod_{p \mid n}(1-1 / p) .
\end{array}
$$

Combining (23) and (24) establishes (22).

A second probabilistic approach to (22) involves an experiment (referred to as the Euler phi process) based on the Riemann zeta process of section 5. Its description follows:

(a) If $p_{l}$ denotes the largest prime less than or equal to $n$, then the array is to have $l$ columns in which the $i^{\text {th }}$ has $p_{i}$ cells. 
(b) To begin, a single solid dot is Bernoulli propelled up each column corresponding to a prime factor of $n$. A column in which a solid dot fails to stop in the top cell is excluded from further consideration.

(c) Set $\mathcal{N}$ equal to $p_{1}^{\alpha_{1}} p_{2}^{\alpha_{2}} \cdots p_{l}^{\alpha_{l}}$ where $\alpha_{i}$ is the number of solid dots that have come to rest in the top cell of column $i$. As the process runs, $\mathcal{N}$ is to be continually updated.

(d) Next, let $c_{1}<c_{2}<\ldots<c_{m}$ denote the columns not excluded in (b). Beginning with $c_{1}$ and proceeding in order, Bernoulli propel a sequence of solid dots up each column; the sequence in column $c_{i}$ is terminated after a solid dot fails to stop in the $p_{c_{i}}^{t h}$ (top) cell or before a hit in the $p_{c_{i}}^{\text {th }}$ cell would result in $\mathcal{N}$ exceeding $n$.

To illustrate, suppose $n=2 \cdot 5$. If the sequences of Bernoulli trials TH and $\mathrm{H}$ occur in step (b), then the result is as pictured on the left in Example 5. Column 3 is excluded from further consideration and $\mathcal{N}=2$.

\section{Example 5.}
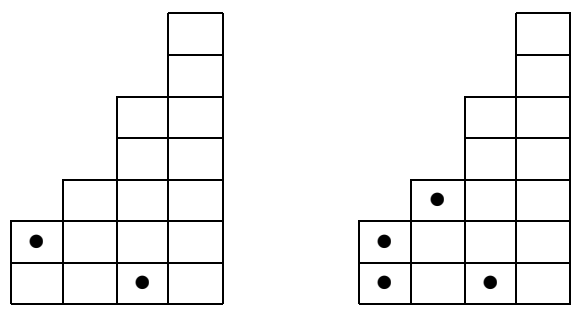

If the next Bernoulli sequences are $\mathrm{H}$ and $\mathrm{TTH}$, then the process terminates as on the above right. The final value of $\mathcal{N}$ is 6 .

Let $\Phi_{n}\{\cdot\}$ denote the measure induced on $\{1,2, \ldots, n\}$ by the Euler phi process. For $1 \leq k \leq n, \Phi_{n}\{\mathcal{N}=k\}$ is readily calculated from (4). Relative to Example 5,

$$
\Phi_{10}\{\mathcal{N}=6\}=\frac{q}{[2]}\left(1-\frac{q^{4}}{[5]}\right)\left(1-\frac{q}{[2]}\right) \frac{q^{2}}{[3]}=\frac{q^{3}}{\|6\|}\left(1-\frac{q^{4}}{[5]}\right)\left(1-\frac{q}{[2]}\right) .
$$

As $q \rightarrow 1^{-}$, the limiting distribution of the Euler phi process for $n=2 \cdot 5$ is illustrated by the probability tree in Figure 1 . The value of $\mathcal{N}$ is computed by multiplying the nodes representing an outcome. Since $\lim _{q \rightarrow 1^{-}} \Phi_{n}\{\cdot\} \neq U_{n}\{\cdot\}$, $\Phi_{n}\{\cdot\}$ is not a $q$-analog of the uniform measure. Nonetheless, $\Phi_{n}\{\cdot\}$ provides the context for the following $q$-analog of (22). As will become evident,

$$
\lim _{q \rightarrow 1^{-}} \Phi_{n}\{\operatorname{gcd}(\mathcal{N}, n)=1\}=U_{n}\{\operatorname{gcd}(\mathcal{N}, n)=1\}=\phi(n) / n
$$

Theorem 5. For $n \geq 1$, define $\phi_{q}(n)=\|n\| \Phi_{n}\{\operatorname{gcd}(\mathcal{N}, n)=1\}$. Then

$$
\lim _{q \rightarrow 1^{-}} \phi_{q}(n)=\phi(n) \quad \text { and } \quad \phi_{q}(n)=\|n\| \prod_{p \mid n}\left(1-q^{p-1} /[p]\right) .
$$

Proof. Suppose that $n=r_{1}^{\alpha_{1}} r_{2}^{\alpha_{2}} \cdots r_{j}^{\alpha_{j}}$, where $r_{1}, r_{2}, \ldots, r_{j}$ are distinct primes and $\alpha_{1}, \alpha_{2}, \ldots, \alpha_{j} \geq 1$. For $1 \leq i_{1}<i_{2}<\ldots<i_{l} \leq j$, it is evident from step (b) in the Euler phi process that

$$
\Phi_{n}\left\{r_{i_{\nu}} \mid \mathcal{N}, 1 \leq \nu \leq l\right\}=\frac{q^{r_{i_{1}}-1} q^{r_{i_{2}}-1} \cdots q^{r_{i_{l}}-1}}{\left[r_{i_{1}}\right]\left[r_{i_{2}}\right] \cdots\left[r_{i_{l}}\right]} .
$$




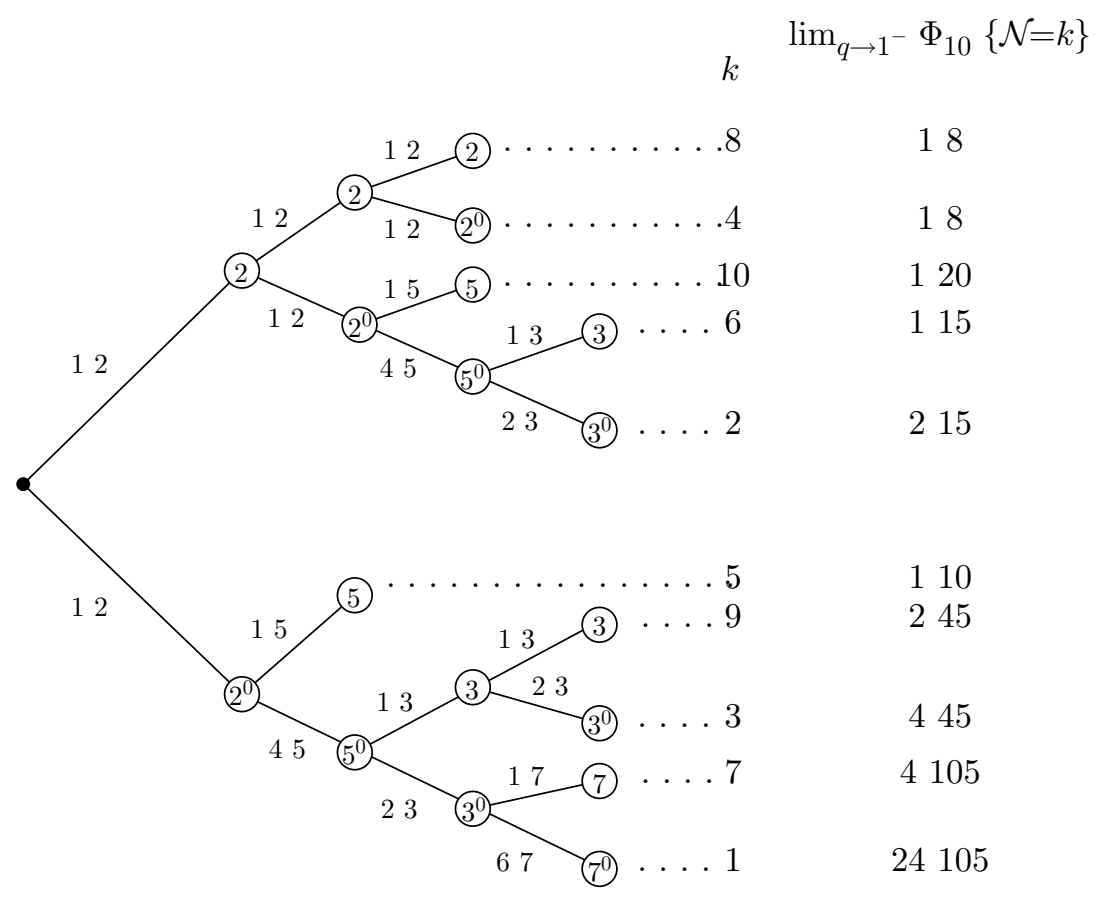

Figure 1

Thus, the events $r_{1}\left|\mathcal{N}, r_{2}\right| \mathcal{N}, \ldots, r_{j} \mid \mathcal{N}$ are independent, and we have

$$
\begin{aligned}
\Phi_{n}\{\operatorname{gcd}(\mathcal{N}, n)=1\} & =\Phi_{n}\left\{r_{i} \backslash \mathcal{N}, 1 \leq i \leq j\right\} \\
& =\prod_{i=1}^{j}\left(1-\Phi_{n}\left\{r_{i} \mid \mathcal{N}\right\}\right)=\prod_{p \mid n}\left(1-q^{p-1} /[p]\right) .
\end{aligned}
$$

Identity (26) implies (25) and the theorem.

\section{Concluding Remark}

Related processes were used in [14] to give probabilistic proofs of identities for Gaussian polynomials, basic hypergeometric series, and Mahonian statistics.

\section{REFERENCES}

1. G. E. Andrews, The Theory of Partitions, Addison-Wesley, 1976. MR 58:27738

2. L. Benkherouf and J. A. Bather, Oil exploration: sequential decisions in the face of uncertainty, J. Appl. Prob. 25 (1988) 529-543. MR 89e:90068

3. F. N. David and D. E. Barton, Combinatorial Chance, Hafner Publ. Co., 1962. MR 27:5305

4. A. M. Garsia and J. Remmel, A combinatorial interpretation of $q$-derangement and $q$-Laguerre numbers, European J. Combin. 1 (1980) 47-59. MR 81g:05011

5. I. Gessel, Counting permutations by descents, greater index, and cycle structure, unpublished manuscript, 1981.

6. I. Gessel and C. Reutenauer, Counting permutations with given cycle structure and descent set, J. Combin. Theory Ser. A 64 (1993) 189-215. MR 95g:05006

7. K. Griffin, The $q$-derangement problem relative to the inversion number, in preparation.

8. W. Feller, An Introduction to Probability Theory and Its Applications, Vol. 1, John Wiley and Sons, 1968. MR 37:3604 
9. N. L. Johnson, S. Kotz, and A. W. Kemp, Univariate Discrete Distributions, John Wiley and Sons Inc., 1992. MR 95d:62018

10. K. W. J. Kadell, A probabilistic proof of Ramanujan's ${ }_{1} \psi_{1}$ sum, SIAM J. Math. Anal. (18) 6 (1987) 1539-1548. MR 88k:33001

11. A. W. Kemp, Heine-Euler extensions of the Poisson distribution, Comm. Statist. Theory and Methods 21 (1992) 571-588. CMP 92:15

12. R. H. Moritz and R. C. Williams, A coin-tossing problem and some related combinatorics, Math. Mag. 61 (1988) 24-29.

13. D. P. Rawlings, Bernoulli trials and number theory, Amer. Math. Monthly 101 (1994), 948-952. MR 95m: 11029

14. D. P. Rawlings, Absorption processes: Models for $q$-identities, Adv. Appl. Math. 18 (1997), 133-148. CMP 97:07

15. D. P. Rawlings and J. A. Treadway, Bernoulli trials and Mahonian statistics: A tale of two q's, Math. Mag. 67 (1994), 345-354. MR 96a:05005

16. M. L. Wachs, On q-derangement numbers, Proc. Amer. Math. Soc. 106 (1989) 273-278. MR 89i:05032

Department of Mathematics, California Polytechnic State University, San Luis OBispo, CALifornia 93407

E-mail address: drawling@math.calpoly.edu 